\title{
Determination of tangential momentum accommodation coefficient and slip coefficients for rarefied gas flow in a microchannel
}

\author{
VADIRAJ HEMADRI ${ }^{1,2}$, AMIT AGRAWAL $^{1, *}$ and U V BHANDARKAR ${ }^{1}$ \\ ${ }^{1}$ Department of Mechanical Engineering, Indian Institute of Technology Bombay, Powai, Mumbai 400076, \\ India \\ ${ }^{2}$ Present Address: Department of Mechanical Engineering, BITS Pilani, Goa Campus, Goa, India \\ e-mail: amit.agrawal@iitb.ac.in
}

MS received 28 July 2017; revised 10 February 2018; accepted 18 April 2018; published online 24 August 2018

\begin{abstract}
This paper presents an experimental study of rarefied gas flow in a trapezoidal microchannel with a constant depth of $103 \mu \mathrm{m}$, top width of $1143 \mu \mathrm{m}$, bottom width of $998 \mu \mathrm{m}$ and length of $2 \mathrm{~cm}$. The aim of the study is to verify the upper limit of the validity of the second-order slip boundary condition to model rarefied gas flows. The slip coefficients and the tangential momentum accommodation coefficient (TMAC) are determined for three different gases, viz. argon, nitrogen and oxygen, and it is observed that they compare well to the literature values. The range of mean Knudsen number $\left(K n_{m}\right)$ investigated is $0.007-1.2$. The non-dimensional mass flow rate exhibits the well-known Knudsen minimum in the transition regime $\left(K n_{m} \sim 1\right)$. It is seen that the Navier-Stokes equation with a second-order boundary condition fits the data satisfactorily with a high value of correlation coefficient $\left(r^{2}>99.95 \%\right)$ in the entire range of $K n_{m}$ investigated. This work contributes by extending the range of Knudsen number studied in the context of validity of the second-order slip boundary condition.
\end{abstract}

Keywords. Slip flow; transition flow; Knudsen minima; tangential momentum accommodation coefficient; second-order slip model.

\section{Introduction}

Fluid flow in microchannels has emerged as an important area of research with the advent of Micro-Electro-Mechanical-Systems (MEMS). Research in the field of micromechanics, in the context of MEMS, started in the late 1980s [1]. Since early 2000, there has been a rapid development in the application of microflow devices in several scientific fields. The design of such devices requires a good understanding of fluid dynamics and heat transfer interactions at the microscale. Rarefaction is a commonly encountered phenomenon in microscale systems involving gases, and plays a vital role in dictating the heat transfer and fluid-flow characteristics of the gas. The rarefaction is quantified by a non-dimensional parameter known as the Knudsen number $(K n)$ defined as the ratio of the mean free path of the gas $(\lambda)$ to the characteristic length of the system $(L)(K n=\lambda / L)$.

In general, for conventional systems, the length scales of the flow are normally much higher than the mean free path of the gas under atmospheric conditions. However, in microfluidic or nanofluidic systems, the characteristic length scale is small and $K n$ can be of order unity even at atmospheric conditions. As the value of Knudsen number increases $(K n>0.1)$, rarefaction effects become more

*For correspondence important and the flow properties associated with the fluid, such as pressure drop, shear stress, heat flux and mass flow rate, cannot be predicted by conventional models based on the continuum hypothesis. Therefore, appropriate corrections (such as the use of velocity slip and temperature jump boundary conditions) are to be made in the Navier-Stokes equations or different approximations may be obtained that simplify the collision integral of the Boltzmann equation (e.g., S-model, BGK model, Liu model) in order to predict the behaviour of flows with high $K n$ [2]. The modelling of flow can be done by assuming the fluid to be either continuous (continuum model) or a collection of discrete molecules (molecular model). The choice of model to be used depends upon the nature of flow being studied, as additional parameters like velocity/temperature slip become significant under rarefied conditions. In the present study, the Navier-Stokes equations with second-order slip coefficients are employed to analyse rarefied gas flow in a trapezoidal microchannel. A general methodology adopted to obtain the velocity slip coefficients (appearing in the velocity slip boundary conditions employed with the Navier-Stokes equations) is to curve-fit the experimental mass flow rate as a function of Knudsen number. The coefficients of the curve-fit can then be utilized to obtain the velocity slip coefficient and the tangential momentum accommodation coefficient (TMAC) [3]. 
Rarefied gas flow has been studied analytically, numerically and experimentally by many authors. The analytical method involves obtaining a solution either starting from the Boltzmann equation, or using the Navier-Stokes with slip boundary conditions to accommodate the rarefaction effects. In [4], the authors used a first-order-accurate slip boundary condition along with 2D Navier-Stokes equation to demonstrate that both compressibility and rarefaction effects are present in long microchannels. Harley et al [5] also applied a first-order boundary condition to predict the friction factor of isothermal, locally fully developed slip flow. The first-order slip boundary condition is commonly employed for slightly rarefied flows $\left(K n_{m} \sim 0.1\right)$. For higher values $\left(K n_{m}>0.1\right)$, the differences between predicted mass flow rates using firstorder model and experimental data are significant. In this framework, several authors have proposed the use of secondorder slip boundary conditions [6, 7]. Sreekanth [8] used the second-order boundary condition in the slip flow regime to predict the mass flow rate and pressure drop for rarefied flow of nitrogen gas through long circular tubes. Beskok and Karniadakis [9] used a second-order slip model and neglected the inertial terms in their analysis. Zohar et al [10] employed perturbation method on the Navier-Stokes equations. They applied a first-order slip boundary condition with full accommodation and $K n_{m}$ of order 0.1. Dongari et al [11] used an integral approach with a second-order boundary condition to study gaseous slip flow in long microchannels. A few authors $[12,13]$ have questioned the validity of the wall-slip assumption and have instead modeled the rarefied flow by including additional diffusion terms in the conventional Navier-Stokes equations.

In experimental studies, mass flow rate is generally the parameter of interest along with the pressure drop across the test section. The most direct method of measuring the mass flow rate is by employing mass flow meters [14]. However, the mass flow meters presently available are limited by their resolution $\left(\sim 10^{-9} \mathrm{~kg} / \mathrm{s}\right)$ and other indirect methods are needed to estimate lower flow rates. They include droplet tracking method [3, 10, 15], constant volume method $[4,7,16]$ and constant pressure method. In the droplet tracking method, the motion of an oil drop induced by the flow is tracked to obtain the volumetric flow rate. This method is however cumbersome to implement and has other disadvantages [7]. These disadvantages can be overcome using the constant volume method or the constant pressure method. The former is usually preferred because of the fabrication difficulties and thermal instability problems associated with the constant pressure method [4]. Maurer et al [3] performed experiments in a shallow microchannel of rectangular cross section from the continuum regime to $K n_{m}$ of 0.6 and 0.8 for nitrogen and helium, respectively. Ewart et al [7] experimentally determined the slip and accommodation coefficients appearing in the solution of the Navier-Stokes equation with second-order slip boundary condition for nitrogen flowing in a microtube. In a further work, Ewart et al [17] performed experiments in a rectangular microchannel with helium as the working fluid and with the experimental range spanning from the continuum regime to the free molecular regime. Their data in the slip regime confirm the value of slip coefficients obtained by Maurer et al [3] under similar conditions.

Although experimental data comparing the slip coefficients in circular and rectangular geometry are available in the literature, such data do not exist for trapezoidal cross section. This cross section is rather typical and obtained by wet etching the microchannel on a silicon wafer of $\langle 100\rangle$ orientation. Trapezoidal cross sections are important because it is observed that the thermal performance of a micro-heat sink with trapezoidal cross section is better than its rectangular counterpart [18, 19]. Furthermore, the data available in the literature largely fall in the slip flow regime $\left(0.001<K n_{m}\right.$ $<0.1)$ and the data in the transition regime $\left(0.1<K n_{m}<10\right)$ is rather sparse. The aim of the present work is to experimentally determine the slip coefficients and TMAC in the slip and transition regimes, to add to the experimental information available on the subject. The other objectives are to verify the applicability of the Navier-Stokes equations with secondorder slip boundary conditions into the early transition regime, to confirm the existence of Knudsen minimum and to determine the influence of cross sectional geometry on the position and magnitude of Knudsen minimum.

\section{Experimental set-up}

A schematic of the experimental set-up designed for obtaining pressure-driven flow is shown in figure 1. The constant-volume method is employed to determine the mass flow rate through the microchannel. This technique uses the pressure variation in the inlet/outlet tank, under quasi-steady-state conditions, to determine the number of moles of gas leaving/ entering the constant volume tanks. The set-up consists of two large reservoirs of volumes $1.3 \times 10^{-3}$ and $4.7 \times 10^{-3} \mathrm{~m}^{3}$, across which a microchannel is connected. The large tank volumes relative to the microchannel ensure that, for low mass flow rates, the pressure variation in the tank is negligible and the quasi-steady-state conditions are valid. The inlet and outlet tanks are connected to the atmosphere and the vacuum pumping system, through a set of valves A-E. Therefore, desired pressures can be imposed on the tanks by venting them either to the atmosphere or the vacuum system.

High-accuracy capacitance manometers (MKS Baratron) (ranges: $0.1,1,10$ and 100 mbar; accuracy $\pm 0.25 \%$ ) are used for pressure measurements at four different locations as shown in figure 1 . The constant-volume approach is well established to measure mass flow rates as low as $10^{-14} \mathrm{~kg} / \mathrm{s}$ [17], well below the lower range offered by commercial mass flow meters $\left(10^{-9} \mathrm{~kg} / \mathrm{s}\right)$. A detailed description of the constant-volume method can be found in many other literatures $[7,16,20]$ and is not included here for brevity.

The microchannel employed in the present measurements was fabricated on a single-side-polished, $2^{\prime \prime}$ silicon wafer. 


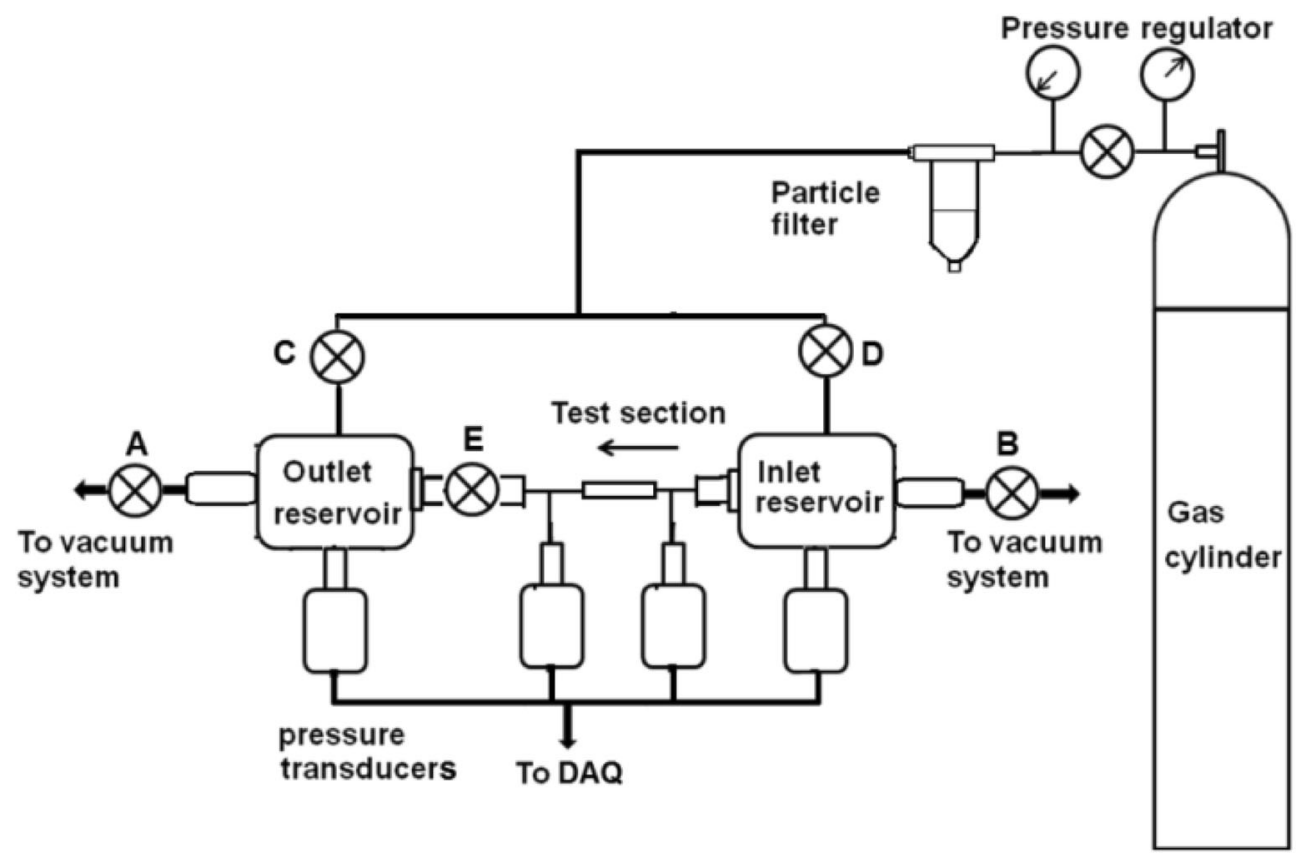

Figure 1. Schematic of the experimental set-up.

The trapezoidal microchannel has a depth of $103 \mu \mathrm{m}$, top width of $1143 \mu \mathrm{m}$, bottom width of $998 \mu \mathrm{m}$ and length of $2 \mathrm{~cm}(H / w=0.09)$. The $\langle 100\rangle$ orientation of the p-type wafer gives the microchannel a trapezoidal cross section after the wet etching process. The top side of the microchannel is covered with an optically smooth quartz plate. Standard lithographic techniques were used for patterning the microchannel. More details of the fabrication process can be found in [21]. The inlet and outlet temperature across the microchannel was measured to verify the isothermal flow assumption. It is observed that for any particular run the temperature variation was within $0.2^{\circ} \mathrm{C}$. The uncertainty in the measured mass flow rate is the total of the uncertainties due to non-isothermal effects $( \pm 2 \%)$, volume of tank $( \pm 1 \%)$ and the standard deviation of the coefficient of linear fit of pressure measurements $(< \pm 1 \%)$. The leakage is estimated as detailed in [22]. Thus, the overall uncertainty in the mass flow rate is evaluated as $\pm 4 \%$.

\section{Theoretical background}

We consider the isothermal rarefied flow of a gas in a microchannel of trapezoidal cross section (figure 2). The channel has a height $H$, length $L$ and width $w$. The theoretical analysis provided in [3] for a rectangular channel is used here with the 'width' of the microchannel replaced by the average of the top and bottom widths, owing to the trapezoidal cross section employed in the present work. A fluid of dynamic viscosity ' $\mu$ ', pressure ' $P$ ' and axial velocity ' $u$ ' flows through the microchannel. Assuming

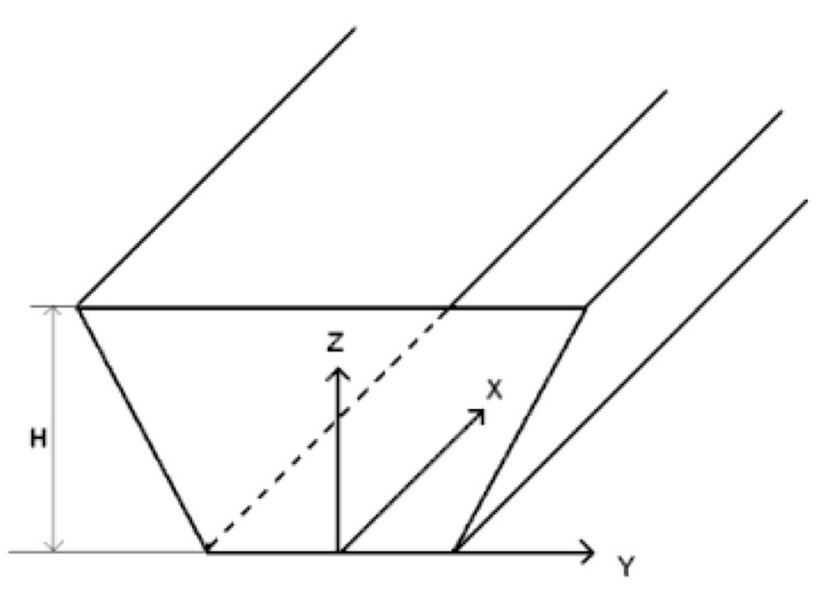

Figure 2. Schematic of the microchannel.

Stokes' approximation and noting that $L \gg w \gg H$, we have

$$
-\frac{\partial P}{\partial x}+\mu\left(\frac{\partial^{2} u}{\partial z^{2}}\right)=0
$$

Since the applicability of the first-order slip boundary condition is limited [3,7], Eq. (1) is solved by employing a second-order slip boundary condition at the upper and lower walls $(z=0$ and $z=H)$ :

$$
u_{w}= \pm \mathrm{C}_{1} \lambda \frac{\partial u}{\partial z}-\mathrm{C}_{2} \lambda^{2} \frac{\partial^{2} u}{\partial z^{2}} .
$$


Here, $C_{1}$ and $C_{2}$ are the slip coefficients. The NavierStokes equation has been used with a second-order slip boundary condition to successfully predict the mass flow rate for $K n_{m}$ up to 0.8 [14]. Solving Eq. (1) with the boundary conditions as prescribed by Eq. (2), we obtain the non-dimensionalized mass flow rate in terms of $K n_{m}$.

$S=\frac{12 \mu R T L \dot{m}}{\Delta P P_{m} w H^{3}}=1+6 \mathrm{C}_{1} K n_{m}+12 \mathrm{C}_{2} K n_{m}^{2} \frac{(\Pi+1) \ln (\Pi)}{(\Pi-1)}$.

In this expression, $R$ is the specific gas constant, $T$ is the absolute temperature, $\dot{m}$ is the mass flow rate, $P_{m}$ is the mean of the inlet and outlet pressures and $\Pi$ is the pressure ratio. The mean Knudsen number is obtained as

$$
K n_{m}=\frac{\mu \sqrt{\pi R T / 2}}{P_{m} H} .
$$

The non-dimensionalized mass flow rate is fitted with a second-order polynomial by the method of least squares:

Table 1. Range of experimental parameters investigated.

\begin{tabular}{lccc}
\hline Gas & Quantity & Min & Max \\
\hline Nitrogen & $P_{m}(\mathrm{~Pa})$ & 63 & 10098 \\
& $K n_{m}$ & 0.0072 & 1.1 \\
& $\dot{m}(\mathrm{~kg} / \mathrm{s})$ & $2 \times 10^{-10}$ & $3.9 \times 10^{-7}$ \\
& $\Pi$ & 7 & 10 \\
Argon & $P_{m}(\mathrm{~Pa})$ & 56 & 12517 \\
& $K n_{m}$ & 0.007 & 1.2 \\
& $\dot{m}(\mathrm{~kg} / \mathrm{s})$ & $2.1 \times 10^{-10}$ & $5.4 \times 10^{-7}$ \\
\multirow{3}{*}{ Oxygen } & $\Pi$ & 6 & 10 \\
& $P_{m}(\mathrm{~Pa})$ & 52 & 10912 \\
& $K n_{m}$ & 0.0072 & 1.13 \\
& $\dot{m}(\mathrm{~kg} / \mathrm{s})$ & $1.8 \times 10^{-10}$ & $4.3 \times 10^{-7}$ \\
& $\Pi$ & 7 & 10 \\
\hline
\end{tabular}

$$
S=1+\mathrm{A} K n_{m}+\mathrm{B} K n_{m}^{2}
$$

where, $A=6 C_{1}$ and $B=12 \mathrm{C}_{2} \frac{(\Pi+1) \ln (\Pi)}{(\Pi-1)}$.

From the determination of $C_{1}$, we can obtain the TMAC using the following relation [3]:

$$
C_{1}=\frac{2}{\sigma}-1 \text {. }
$$

Loyalka [23] obtained the 'exact' numerical solution of the BGK model and proposed the following expression for the calculation of the accommodation coefficient:

$$
C_{1}=\frac{\sqrt{\pi} 2}{2} \frac{2-\sigma}{\sigma}(1+0.1621 \sigma) .
$$

As seen from Eq. (3), the non-dimensionalized mass flow rate can be expressed in terms of the mean Knudsen number and the pressure ratio. Therefore, it is possible to experimentally obtain the slip coefficients by curve fitting the non-dimensional mass flow rate with the expression provided in Eq. (3). The determination of the slip coefficients is necessary to obtain the slip velocity in the case of rarefied gas flows. This also allows for a comparison of the dependence of the slip coefficients (and hence slip velocity) on different gas-solid interfaces and different cross sections.

\section{Determination of slip coefficients}

The range of experimental parameters is tabulated in table 1. Figure $3 a, b$ and $c$ shows the variation of the nondimensional mass flow rate $S$ with the mean Knudsen number for three different gases (argon, nitrogen and oxygen, respectively). In all the three cases, the non-dimensional experimental mass flow rate is fitted with a second-order polynomial using a least squares method. This is done to ascertain the applicability of the Navier-Stokes

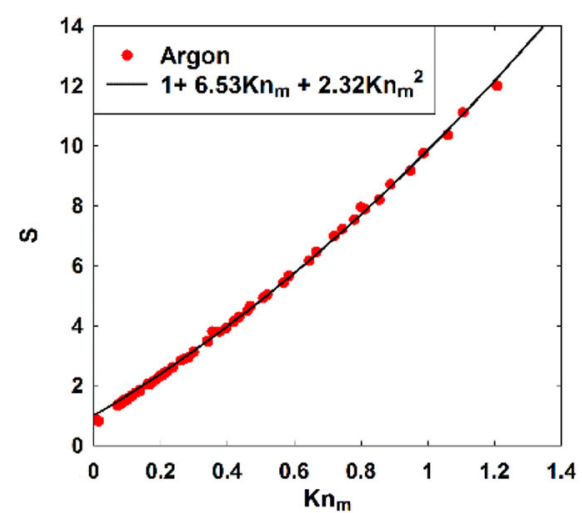

(a)

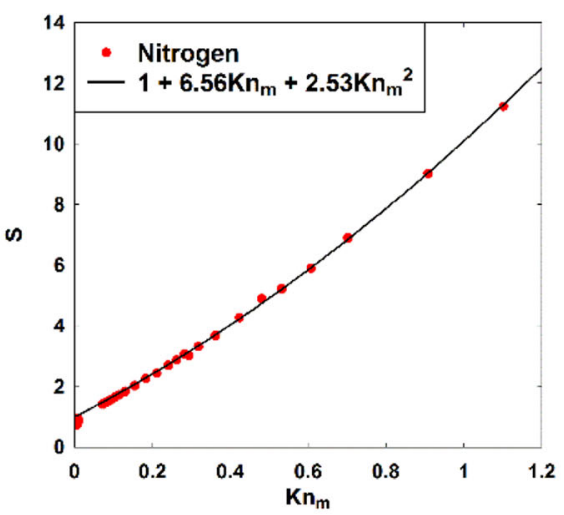

(b)

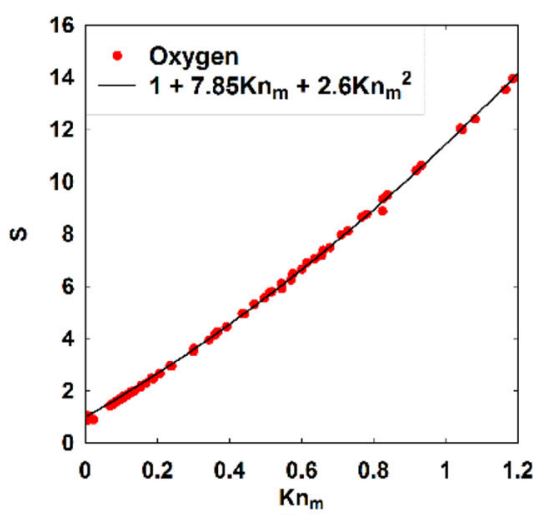

(c)

Figure 3. Experimental non-dimensional mass flow rate fitted with a second-order polynomial for (a) argon, (b) nitrogen and (c) oxygen. 
equation with second-order slip boundary condition in predicting the mass flow rate for $K n_{m}>0.3$. It is seen that the second-order fit matches the experimental data with a high value of correlation coefficient $\left(r^{2}>99.95 \%\right)$ over the entire range of $K n_{m}$ encountered in the experiments.

The slip coefficients are determined by Eq. (5) and subsequently, the TMAC is determined in two ways: through the application of Eqs. (6) and (7) for different gases and tabulated in table 2 along with those reported in the literature for other cross sections. Since the pressure ratio varies during the experiments, in the determination of $C_{2}$ from Eq. (5), the average pressure ratio is used. The uncertainties on the regression coefficients are obtained using the standard error analysis. It is seen from table 2 that the second-order coefficients of the present work and Maurer et al [3] are close to each other. This is due to the higher range of Knudsen numbers covered in these experiments. In contrast, the second-order coefficients of Yamaguchi et al [24] are negligible and the data are mostly linear due to the low range of Knudsen numbers in their experiments. However, the TMAC obtained in the present experiments is seen to be higher than those obtained by Maurer et al [3] and Yamaguchi et al [24].

\section{Effect of cross section}

Figure 4 shows a comparison of non-dimensional mass flow rate for different cross sections and gases. It is seen that for rectangular and trapezoidal cross sections considered, respectively, in [3] and the present work (in both cases $H<<w$ ), the effect of lateral walls is negligible and the data agree well over the entire Knudsen number range studied. Although the present study considered a trapezoidal cross section that has not been studied earlier, it is seen that the effect of cross section is not very evident due to the low aspect ratio considered here. Nevertheless, this study is useful as it considers a realistic cross section that is typically encountered due to microfabrication of microchannels.

The data plotted for circular tube [24] show a higher value of $S$ and is also a stronger function of Knudsen

Table 2. Slip coefficients and TMAC for different gases.

\begin{tabular}{lcccccc}
\hline Gas & & & & TMAC & TMAC & \\
An $n_{m}$ range & $C_{1}$ & $C_{2}$ & (Eq. (6)) & (Eq. (7)) & \\
\hline Argon & $0.07-1.2$ & $1.089 \pm 0.08$ & $0.140 \pm 0.007$ & $0.957 \pm 0.04$ & $0.970 \pm 0.03$ & Present work (trapezoidal) \\
Nitrogen & $0.072-0.11$ & $1.093 \pm 0.07$ & $0.151 \pm 0.003$ & $0.955 \pm 0.03$ & $0.968 \pm 0.03$ & \\
Oxygen & $0.072-1.13$ & $1.301 \pm 0.09$ & $0.155 \pm 0.004$ & $0.866 \pm 0.04$ & $0.874 \pm 0.03$ & \\
Argon & $0.05-0.3$ & $1.30 \pm 0.09$ & $0.063 \pm 0.018$ & $0.869 \pm 0.03$ & $0.872 \pm 0.03$ & Yamaguchi et al [24] (circular) \\
Nitrogen & $0.05-0.3$ & $1.35 \pm 0.06$ & $0.031 \pm 0.005$ & $0.851 \pm 0.02$ & $0.851 \pm 0.02$ & \\
Oxygen & $0.05-0.3$ & $1.35 \pm 0.06$ & $0.028 \pm 0.004$ & $0.851 \pm 0.02$ & $0.851 \pm 0.02$ & Maurer et al [3] (rectangular) \\
Helium & $0.006-0.8$ & $1.2 \pm 0.05$ & $0.23 \pm 0.1$ & $0.91 \pm 0.03$ & $0.915 \pm 0.03$ & \\
Nitrogen & $0.006-0.7$ & $1.3 \pm 0.05$ & $0.26 \pm 0.1$ & $0.87 \pm 0.03$ & $0.872 \pm 0.03$ & \\
\hline
\end{tabular}

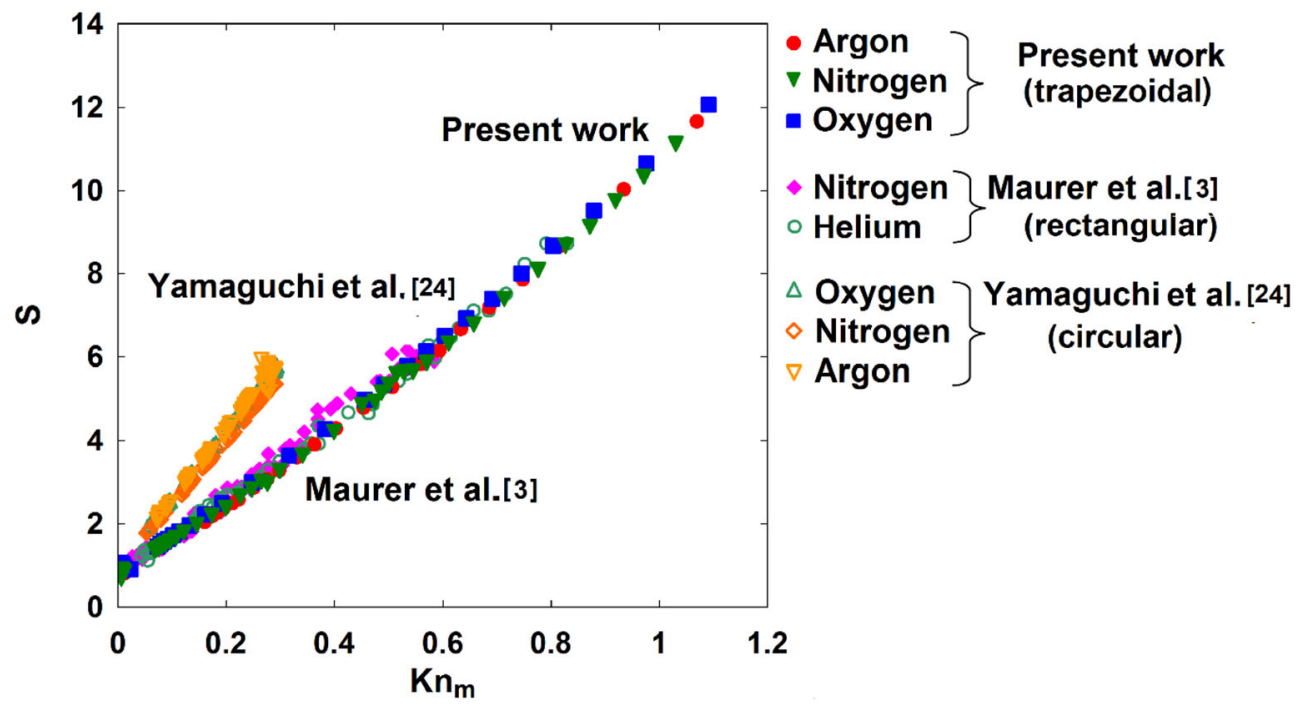

Figure 4. Comparison of non-dimensional mass flow rates across different studies involving different gases and cross sectional geometries. 
number as compared with both rectangular and trapezoidal microchannels. For the range of Knudsen number studied here, the effect of molecular weight of the gas is seen to be absent, as the data points for different gases converge to a single curve. Comparing the present results directly to theory is difficult for a variety of reasons. Firstly, many authors have obtained expressions for non-dimensional mass flow rates by treating the rarefaction parameter to be constant along the channel. In our case, the pressure ratio is considerably large ( $\sim 7$ to 10$)$ and the local pressure variations have not been determined.

\section{Knudsen minimum}

As seen from table 2, the pressure ratios encountered in the present set of experiments are very high (ranging from 7 to 10). In such cases, the non-dimensionalization of mass flow rate based on the mean pressure is inappropriate, as the gas rarefaction varies considerably along the length of the microchannel. Following the approach adopted by Sharipov [25] and Ewart et al [17] for rarefied flow in long microchannels with high pressure ratio, the mass flow rate is non-dimensionalized as

$$
G=\frac{L \sqrt{2 R T}}{H^{2} w\left(P_{i}-P_{o}\right)} \dot{m} .
$$

The reduced mass flow rate expressed as shown here is independent of the local pressure and becomes a function of the inlet and outlet pressures only. This non-dimensionalized mass flow rate is expected to exhibit the Knudsen minimum phenomenon, which is an important characteristic of rarefied gas flows. The variation of the reduced mass flow rate $G$ with $K n_{m}$ for the three different gases is plotted in figure 5 .

It is seen that the Knudsen minimum is observed around a mean Knudsen number of slightly less than unity in all the studies. For comparison, we use the expression for the reduced mass flow $(G)$ rate provided by [25] for a rectangular cross section with $H / w=0.1$ and fully diffuse reflection at the boundary (TMAC $=1$ ). The present data agree well to that of [25] at lower $K n_{m}$. As $K n_{m}$ increases, the predicted results of [25] deviate from the experimental results (figure 5, maximum deviation is $16 \%$ ). This indicates that the molecular reflection at the wall is not entirely diffusive in nature for $K n_{m}>0.4$, since the theoretical model is based on fully diffuse accommodation. Agrawal and Prabhu [26] suggested a TMAC value of 0.93 for monatomic gases for the whole Knudsen number range based on a survey of data in the literature. The experimental value obtained in the present work for argon $(0.957 \pm 0.04)$ is in agreement to the value suggested from literature. The experimental results of Ewart et al [17] for a rectangular cross section are also in good agreement to the present set of results, which are carried out for a trapezoidal cross section. A quantitative agreement among rectangular and trapezoidal cross section indicates

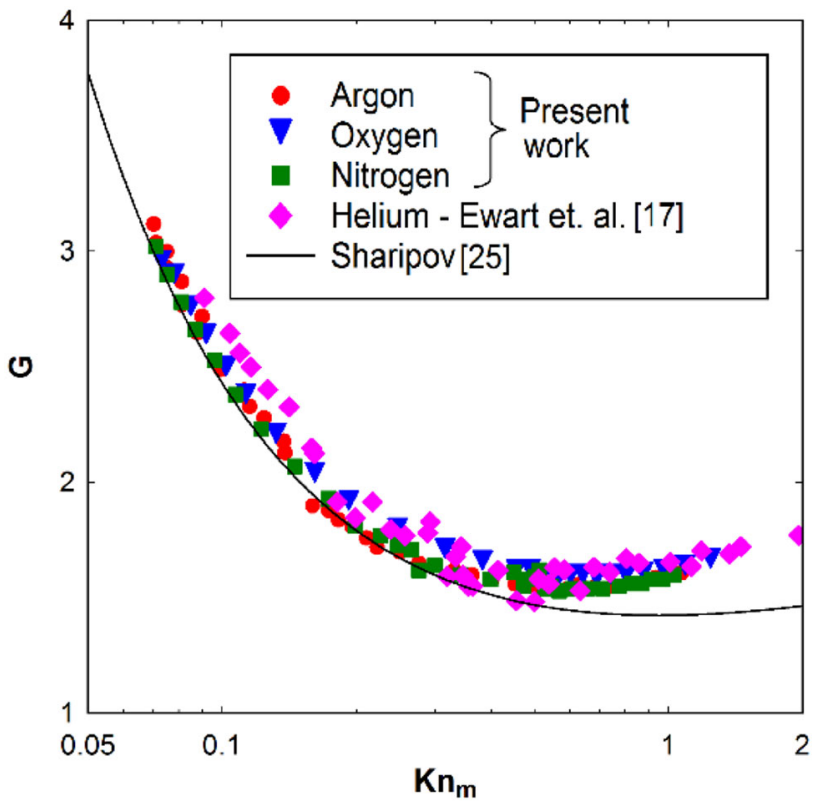

Figure 5. Comparison of non-dimensional mass flow rate obtained in different studies.

negligible effect of the lateral walls for the low aspect ratio considered in these studies $(H / w<0.1)$.

Till recently, there was no clarity on the position and magnitude of Knudsen minimum (or even its existence) for microchannels of non-uniform cross section. Recent measurements by Hemadri et al [27, 28] have experimentally established the existence of Knudsen minimum in diverging and converging microchannels. They also noted the position and magnitude of the minimum to be weakly dependent on the geometrical cross section of the microchannel and the molecular weight of the gas species. The effect of side walls was seen to be negligible as gas flow in converging/diverging microchannels approached the parallel plate solution for low $K n_{m}$.

\section{Conclusions}

This paper presented an experimental study to verify the validity of the Navier-Stokes equations with second-order boundary conditions in a microchannel of trapezoidal cross section. The slip coefficients and TMAC were obtained for three different gases and it was shown that a second-order boundary condition can successfully predict the mass flow rate up to $K n_{m} \sim 1$. These experimentally obtained slip coefficients are significant because they form a part of the database that will help identify the effect of various parameters such as gas-solid interaction, surface roughness and microchannel geometry on rarefied gas flows. The extension of the use of Navier-Stokes equation with slip boundary condition is desirable, due to the high cost involved in simulating flows at high Knudsen numbers (slip 
and transition regimes) using alternate techniques such as direct simulation Monte Carlo.

The Knudsen minimum is observed in the present experiments at $K n_{m} \sim 1$. The position and magnitude of the Knudsen minimum is seen to be unaffected by the molecular weight of the gas. Moreover, the Knudsen minimum for the trapezoidal cross section microchannel quantitatively agrees with the data for rectangular microchannel, implying negligible influence of the side lateral walls on the flow behaviour for low-aspect-ratio microchannels.

\section{References}

[1] Karniadakis G E and Beskok A 2002 Microflows-fundamentals and simulations. New York: Springer

[2] Gad-el-Hak M 1999 The fluid mechanics of microdevicesthe Freeman scholar lecture. J. Fluids Eng. 121: 5-33

[3] Maurer J, Tabeling P, Joseph P and Willaime H 2003 Second-order slip laws in microchannels for helium and nitrogen. Phys. Fluids 15: 2613-2621

[4] Arkilic E B, Schimidt M A and Breuer K S 1997 Gaseous slip flow in long micro-channels. J. Microelectromech. Syst. 6: $167-178$

[5] Harley J, Huang Y, Bau H and Zemel J N 1995 Gas flow in microchannels. J. Fluid Mech. 284: 257-274

[6] Colin S, Lalonde P and Caen R 2004 Validation of a secondorder slip flow model in rectangular microchannels. Heat Transf. Eng. 25: 23-30

[7] Ewart T, Perrier P, Graur I A and Meolans J G 2006 Mass flow rate measurements in gas microflows. Exp. Fluids 41: 487-498

[8] Sreekanth A K 1969 Slip flow through long circular tubes. In: Proceedings of the Sixth International Symposium on Rarefied Gas Dynamics, pp. 667-680

[9] Beskok A and Karniadakis G E 1999 A model for flows in channels, pipes, and ducts at micro and nano scales. Microsc. Thermophys. Eng. 3: 43-77

[10] Zohar Y, Lee S Y K, Lee W Y, Jiang L and Tong P 2002 Subsonic gas flow in a straight and uniform microchannel. $J$. Fluid Mech. 472: 125-151

[11] Dongari N, Agrawal A and Agrawal A 2007 Analytical solution of gaseous slip flow in long microchannels. Int. J. Heat Mass Transf. 50: 3411-3421

[12] Chakraborty S and Durst F 2007 Derivations of extended Navier-Stokes equations from upscaled molecular transport considerations for compressible ideal gas flows: towards extended constitutive forms. Phys. Fluids 19: 088104

[13] Dongari N, Sharma A and Durst F 2008 Pressure-driven diffusive gas flows in micro-channels: from the Knudsen to the continuum regimes. Microfluid. Nanofluid. 6: 679-692

[14] Sreekanth A K 1965 Transition flow through short circular tubes. Phys. Fluids 8: 1951-1956

[15] Harley J, Huang Y, Bau H and Zemel J N 1995 Gas flow in microchannels. J. Fluid Mech. 284: 257-274

[16] Pitakarnnop J, Varoutis S, Valougeorgis D, Geoffroy S, Baldas L and Colin S 2010 A novel experimental setup for gas microflows. Microfluid. Nanofluid. 8: 57-72

[17] Ewart T, Perrier P, Graur I A and Meolans J G 2007 Mass flow rate measurements in a microchannel, from hydrodynamic to near free molecular regimes. J. Fluid Mech. 584: 337-356

[18] Morini G L, Lorenzini M, Salvigni S and Spiga M 2006 Thermal performance of silicon micro heat-sinks with electrokinetically-driven flows. Int. J. Therm. Sci. 45: 955-961

[19] Han Y, Lee Y J and Zhang X 2013 Trapezoidal microchannel heat sink with pressure-driven and electro-osmotic flows for microelectronic cooling. IEEE Trans. Compon. Packaging Manuf. Technol. 3: 1851-1858

[20] Perrier P, Graur I A, Ewart T and Meolans J G 2011 Mass flow rate measurements in microtubes: from hydrodynamic to near free molecular regime. Phys. Fluids 23: 042004

[21] Singh S G, Kulkarni A, Duttagupta S P, Puranik B P and Agrawal A 2008 Impact of aspect ratio on flow boiling of water in rectangular microchannel. Exp. Therm. Fluid Sci. 33:153-160

[22] Demsis A, Verma B, Prabhu S V and Agrawal A 2010 Heat transfer coefficient of gas flowing in a circular tube under rarefied condition. Int. J. Therm. Sci. 49: 1994-1999

[23] Loyalka S 1975 Kinetic theory of thermal transpiration and mechanocaloric effect II. J. Chem. Phys. 63: 4054

[24] Yamaguchi H, Hanawa T, Yamamoto O, Matsuda Y, Egami $\mathrm{Y}$ and Niimi T 2011 Experimental measurement on tangential momentum accommodation coefficient in a single microtube. Microfluid. Nanofluid. 11: 57-64

[25] Sharipov F 1999 Rarefied gas flow through a long rectangular channel. J. Vac. Sci. Technol. A 17: 3062-3066

[26] Agrawal A and Prabhu S V 2008 Survey on measurement of tangential momentum accommodation coefficient. J. Vac. Sci. Technol. A 26: 634-645

[27] Hemadri V, Varade V V, Agrawal A and Bhandarkar U V 2016 Investigation of rarefied gas flow in microchannels of non-uniform cross section. Phys. Fluids 28: 022007

[28] Hemadri V, Varade V V, Agrawal A and Bhandarkar U V 2017 Rarefied gas flow in converging microchannel in slip and early transition regimes. Phys. Fluids 29: 032002 